\title{
Design of Servo Motor Control System Based on POWERLINK Fieldbus
}

\author{
Dang-feng Pang, Jiang Wang \\ School of Electrical Engineering and Automation, Tianjin \\ University Tianjin,China \\ E-mail: tjdangfeng@163.com
}

\author{
Dang-Feng Pang, Li-Qiang Wang, Sheng-Li Lu \\ Tianjin University of Technology and Education \\ Tianjin,China \\ E-mail: tjdangfeng@163.com
}

\begin{abstract}
With the continuous development of science and technology, the servo motor control system based on real time industrial Ethernet is now more widely applied in the field of industrial automation than ever. This paper expounds the design of a servo motor control system based on POWERLINK Industrial Ethernet. The system is proven to be highly reliable and stable due to the open source and good real-time performance characteristics of the POWERLINK.
\end{abstract}

Keywords-POWERLINK bus; industrial ethernet; servo motor control

\section{INTRODUCTION}

Fieldbus technology is a kind of advanced industrial control technology, which applies the computer network and digital communication technology to the industrial control field. Currently in the field of industrial automation field bus standards, there are EtherNet/IP, IRT Prolinet, Modbus RTPS EtherCAT., POWERLINK Ethernet. These types of real-time Ethernet technology using the same hardware layer, in accordance with the IEEE802.3 standard, and therefore can use standard Ethernet cable, interface and other hardware facilities, so the real-time Ethernet can and standard Ethernet compatible.

The field bus technology is applied to the servo control system, which can enhance the communication ability between the servo drive device and the control unit. In this paper, a servo motor control system based on POWERLINK is designed. The system has fast response speed and stability.

\section{POWERLINK INDUSTRIAL ETHERNET}

POWERLINK is a field bus standard in Europe, which has the characteristics of high performance, future, standardization, openness, economy and so on. POWERLINK is a kind of industrial Ethernet which is based on industrial field bus standard interface. It is a kind of high real-time bus technology, which supports standard Ethernet from $10 \mathrm{M}$ to $1000 \mathrm{M}$. At the same time it is a kind of technology open source field bus technology, it is based on the standard Ethernet, no need for a dedicated ASIC chip, no need to purchase authorization, there is a place where Ethernet can be realized. POWERLINK network capacity can support 240 nodes, each node supports 1500Bytes input and 1500Bytes output, the network cycle cycle is only 200us.

The synchronization of POWERLINK bus is high, its cycle is short, at the same time it is convenient for modular design and distributed control, support cross communication and network connection is stable and reliable. POWERLINK provides a unified, open source CAN open code. For POWERLINK users, as a result of the adoption of a unified standard CAN open application layer, to ensure that all POWERLINK devices can interconnect.

An important feature of POWERLINK is cross communication. The Cross communication refers to the free transmission of data from the station without intervention. Because each slave station sends data out is the radio sent out, so all the nodes on the network can receive in this cycle, if a node needs the data, then you can direct charge. POWERLINK supports a variety of redundancy, including multi master redundancy, and the redundant dual ring redundancy.

OSI is a standard communication model for the open information processing system. This model divides the tasks into 7 layers, each layer is built on the basis of the next layer. The POWERLINK network model according to the OSI model in the hierarchy as shown in Figure 1

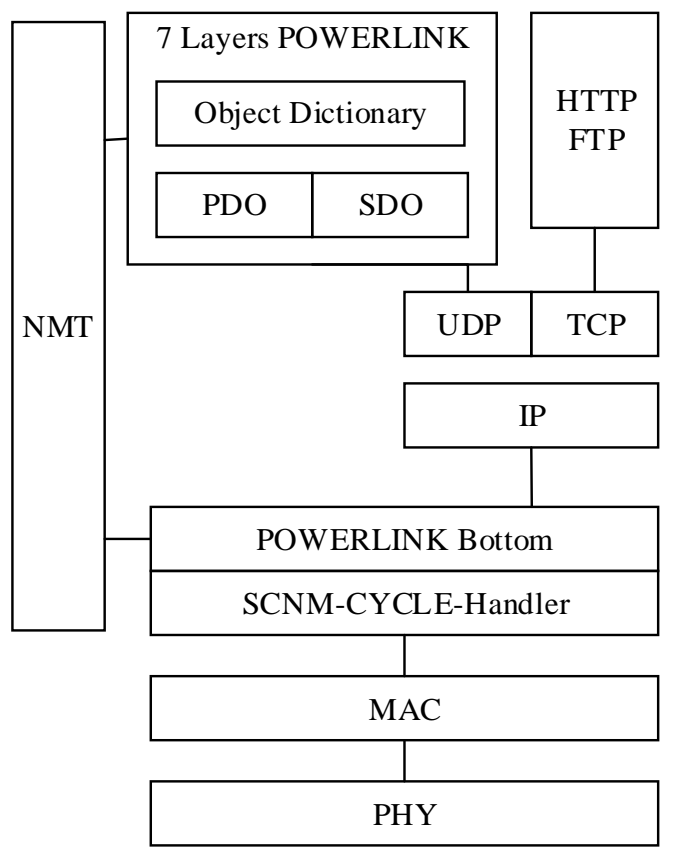

Figure 1. The OSI model of POWERLINK network.

\section{THE STRUCTURE OF SERVO CONTROL SYSTEM}

A typical servo motor control vector control mode, the general choice of the fundamental direction of the permanent magnetic field for the $\mathrm{d}$ axis, $\mathrm{q}$ axis ahead of 90 degrees, its 
speed and synchronous speed is the same. The stator current according to the coordinates of the conversion, as shown in Figure 2, the component Ia and component Iq, Iq used to generate torque. Compared with the simple control method, the Ia is zero, and the three-phase synthetic current IS can be obtained almost all of the torque, so that the torque has the maximum value. The control method of permanent magnet synchronous servo motor driver using Id is zero.

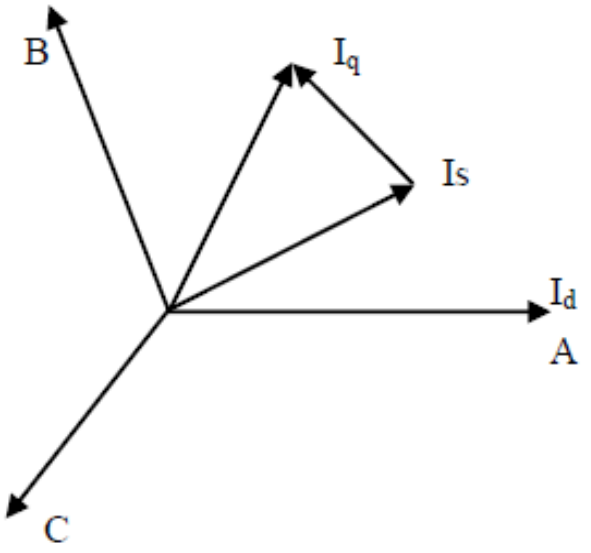

Figure 2. Vector decomposition of servo motor.

$$
\begin{aligned}
& \mathrm{U}_{\mathrm{d}}=\mathrm{P} \Psi_{\mathrm{d}}+\mathrm{RI}_{\mathrm{d}}-\Psi_{\mathrm{q}} \omega \\
& \mathrm{U}_{\mathrm{q}}=\mathrm{P} \Psi_{\mathrm{q}}+\mathrm{RI}_{\mathrm{q}}+\Psi_{\mathrm{d}} \omega \\
& \mathrm{T}_{\mathrm{e}}=\mathrm{n}_{\mathrm{p}}\left(\mathrm{I}_{\mathrm{q}} \Psi_{\mathrm{d}}-\mathrm{I}_{\mathrm{d}} \Psi_{\mathrm{q}}\right)
\end{aligned}
$$

$\mathrm{Ud}$ and Uq express the instantaneous value of the stator voltage decomposition on the Id and Iq axes. P represents a differential operator. $d$ and $q$ expressed on the $d$ and $q$ axis component of the stator link. Theis rotation speed of the dq axis. np represents the pole pairs, Te indicates the output torque[1][2].

Servo control system is mainly composed of controller, servo drive device, servo motor and feedback unit. The network topology is usually composed of linear structure, circular structure, star type structure, daisy chain type, network type, tree type etc.. In this paper, the design of dual servo motor control system using linear network structure.

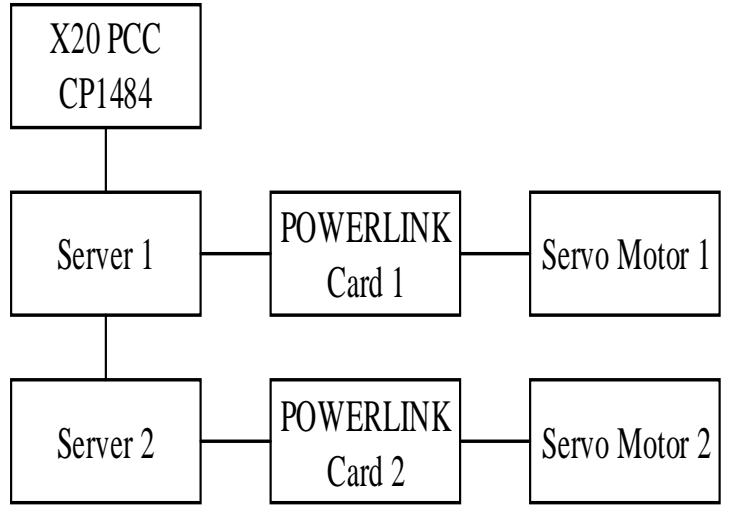

Figure 3. Structure of servo system.
The performance of the servo control system depends on the selection of the control bus. The speed of the bus and the efficiency of the protocol directly affect the data update time of the servo axis, and then affect the control precision of the servo axis. The stability of the control system is determined by the safety and reliability of the bus and the ability of self diagnosis and self recovery.

Servo motor control system using the servo drive model is $8 \mathrm{~V} 1010.00-2 \mathrm{~B} \& \mathrm{R}$ industrial automation company, encoder card model is 8AC122.60-3, POWERLINK card model is 8AC114.60-2, the servo motor model is 8LSA25.R0060D000-3. Master the use of B\&R X20 series PCC, integrated POWERLINK interface, the model is $\mathrm{X} 20 \mathrm{CP} 1484$. CP1484 is a powerful X 20 system CPU, specifically applied to a very short cycle of the project, able to deal with a large number of data, floating-point operations [3].

\section{THE CONTROLLER PROGRAM DESIGN}

Automation studio (ASES) software is B\&R products using a unified development platform for hardware configuration and modular programming. AS software supports IEC61131-3 programming language, Basic language and C language. AS software application is strong, suitable for all kinds of program development staff. In this paper, the standard type controller CP 1484, integrated I/O processor, the minimum cycle period up to $800 \mathrm{us}$, the typical instruction cycle is 0.022 us, the interface IF3 for real time Ethernet POWERLINK. The software environment of the system is AS4.2, which is stable, real-time and optional.

Automation Runtime also provides the same interface to the software developers, developers do not need to consider the details can be directly to the control system hardware structure to achieve management. Automation Runtime in IO management, the $\mathrm{I} / \mathrm{O}$ module signal is transmitted to the mapping table before the start of the task, and then the output signal is transferred to the mapping table after the end of the task scan cycle. I/O mapping table will be the task of the process variables and the $\mathrm{I} / \mathrm{O}$ module input and output mapping directly, in addition to direct the configuration of the special attributes of the I/O module. Automation Runtime memory area is divided into two parts, ram and ROM, ROM in the CF card and is divided into UserROM and SystemROM, project of atherosclerosis and related configuration file is stored in the UserROM, SystemROM is stored automation runtime and called the project file system [4].

The Real time operating system Automation Runtime is an important part of AS software. It is the software kernel that supports the motion control operating system to run on the target system. Through the TCI'/IP network or serial port RS232 users on the AS developed a good servo control data transmission to the target system, Automation Runtime and Windows, Linux and other operating systems such as the implementation of these content[5][6].

After the system is powered on, the master station is automatically activated. Initialization is divided into two parts, that is, the POWERLINK controller initialization and POWERLINK protocol stack initialization. The initialization 
of the controller includes the version, the serial number, the identification code, the MAC address, the product number, and so on. POWERLINK protocol stack of the initial implementation of the successful completion of the system parameters configuration. The servo motor control mode is the first position control, followed by speed control, when final torque control. The master sequence control flow chart is shown in figure 4.

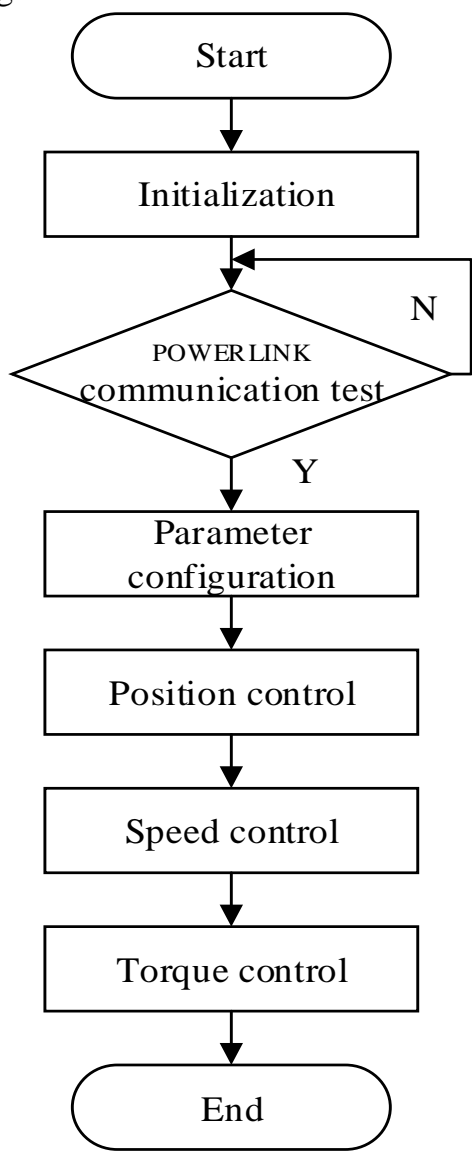

Figure 4. Master sequence control flow chart.

AS software NCTest can be used for servo motor control online debugging, it combines the drive motor analysis and testing of all the features. NCTest includes four parts: NcTrace, NcWatch, parameter windows and command interface. NCWatch shows the current state of the axis, NCTrace records the set of real-time data and the way of the chart shows that the parameters of the window is the corresponding parameters of the operating structure, the command interface can control the axis. So, in NCTest, can control the servo drive through the visual window, you can directly control the $\mathrm{NC}$ action, and display the status information. The NCTest test results are shown in figure 5.

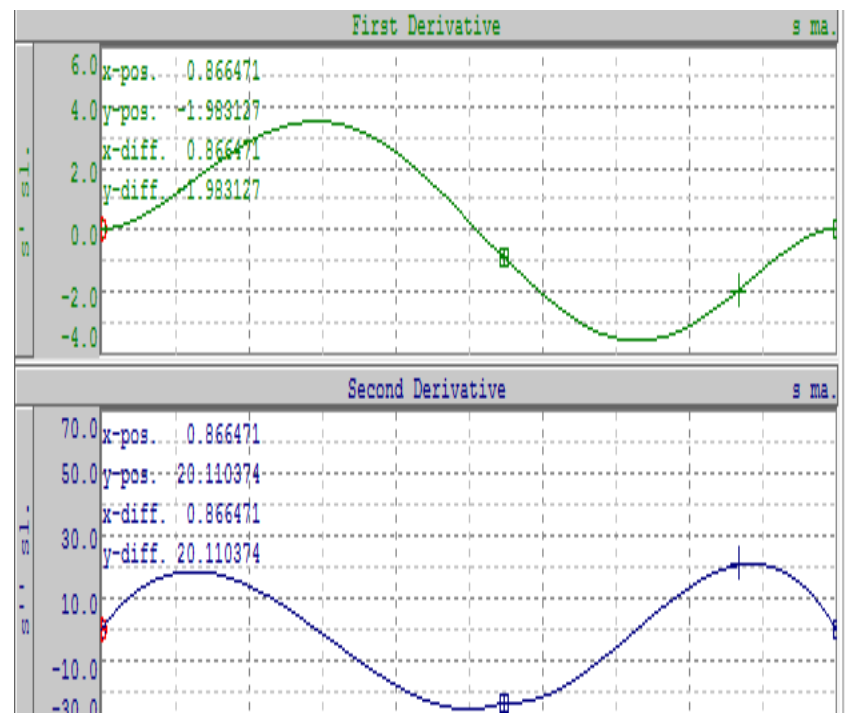

Figure 5. The test results of cam path.

\section{CONCLUSIONS}

This paper studies the principle of operation and POWERLINK Industrial Ethernet communication mechanism of the servo motor control system, and designed a dual servo motor control system based on POWERLINK, applied AS software on the Bei Calais controller for the implementation for the configuration of the control system of servo motor as well as the completed the software design of the system. The system has flexible development, high speed of operation control, and it has a certain application reference value.

\section{ACKNOWLEDGMENT}

This work is supported by 2015 annual cooperation professional comprehensive reform program (Development of motion control teaching case based on POWERLINK bus and Development of modern control technology experience curriculum), all support is gratefully acknowledged.

\section{REFERENCE}

[1] Wang D,The Research of Networked AC Servo System Based on POWERLINK[D],Shan Dong:Shan Dong University.2014.

[2] Xu G.X.The Robot System Based on POWERLINK Fieldbus[D],Shan Dong:Shan Dong University.2015.

[3] Xiao W.R, Wang J.Q, Song H.Z. Explanation of Open Source Real-time Ethernet POWERLINK [M].Beijing: China Machine Press .

[4] Erwinski K, Paprocki M, Grzesiak L M, et al. Application of Ethernet POWERLINK for communication in a Linux open CNC system[J]. IEEE Transactions on, 2013, 60(2): 628-636.

[5] Paul R P, Shimano B E, Mayer G.Kinematic control equations for simple manipulator[J].IEEE Trans SMC,2001(6):449-455.

[6] Schemm E. SERCOS to link with ethernet for its third generation[J]. IEEE Computing and Control Engineering, 2004, 15(2): 30-33. 